\title{
Simultaneous identification and determination of biogenic amines in Pueraria lobata by ultra-high performance liquid chromatography
}

\author{
YuJie $\mathrm{Wu}^{1,2} \mathbb{D} \cdot$ XiangYu Cai $^{2} \cdot$ SiHua Jiang ${ }^{2}$
}

Received: 2 January 2020 / Accepted: 21 August 2020 / Published online: 12 September 2020

(c) Springer Nature Switzerland AG 2020

\begin{abstract}
A method of ultra-performance liquid chromatography (UPLC) with precolumn derivatization has been established for identification and simultaneous determination of nine biogenic amines as tryptamine, phenethylamine, putrescine, cadaverine, histamine, octopamine, tyramine, spermidine and spermine in pueraria lobata. Samples were extracted with perchloric acid solution, derivitazation with dansyl chloride, added ammonia water for termination reaction and then made up to the desired volume with acetonitrile. The chromatographic separation was performed on Acquity UPLC BEH C18 column $(2.1 \mathrm{~mm} \times 100 \mathrm{~mm}, 1.7 \mu \mathrm{m})$ using $5 \mathrm{mmol} / \mathrm{L}$ ammonium acetate and acetonitrile as mobile phase for gradient elution at the flow rate of $0.4 \mathrm{~mL} / \mathrm{min}$. Photo-diode array detector was set at wavelength of $254 \mathrm{~nm}$ for all of the biogenic amines. Nine biogenic amines had good linear relationships in the concentration range of $0.1-20 \mu \mathrm{g} / \mathrm{mL}$ with correlation coefficients larger than 0.999 . The recoveries of samples spiked at levels of 20,50 and $100 \mathrm{mg} / \mathrm{kg}$ were $75.6-97.2 \%$ with the relative standard deviations of $0.12-5.32 \%(n=6)$. The limits of detection of the nine biogenic amines were all $10 \mathrm{mg} /$ $\mathrm{kg}$. The established method is simple, rapid and sensitive, which can be used for simultaneous determination of nine biogenic amines residues in Pueraria lobata and other plant medicine. By the established method, it was the first time to verify that there are biogenic amines in Pueraria lobata all over the world, which would be used to study the health care mechanism of Pueraria lobata for people.
\end{abstract}

Keywords Ultra performance liquid chromatography · Biogenic amine $\cdot$ Simultaneous determination · Pueraria lobata

\section{Introduction}

Biogenic amines belong to low molecular weight alkaline compounds with biological activity and nitrogen, they are mainly formed by corresponding amino acids under microbial decarboxylation, or by aldehydes and ketones under the action of amino acid transaminase [18]. Common biogenic amines include tryptamine, putrescine, tyramine, histamine, spermine, spermidine, phenylethylamine, octapamine, cadaveric amine, etc. [9]. Polyamines play an important role in cell proliferation and differentiation, regulation of nucleic acid function, protein synthesis, brain development, regulation of nerve growth and regeneration $[6,9,13,15]$. Due to the physiological activity of biogenic amines in, the research on biogenic amines has attracted widespread attention. Biogenic amines are widely found in a variety of foods and beverages, especially in protein-rich foods, amino acids, fermented foods [7-12, 19]. However, there are few reports on biogenic amines in Chinese herbal medicine, especially in Pueraria Lobata. As we all know, Pueraria Lobata is a very good treatment of pain and other symptoms of drug and food homologous products in China. In order to study the mechanism of action of Pueraria Lobata, it is necessary to understand the active components of biogenic amines in Pueraria

$\triangle$ YuJie Wu, wyjhappy_2001@163.com | 'Institute of Food Safety, Chinese Academy of Inspection and Quarantine, Beijing 100176, People's Republic of China. ${ }^{2}$ Technology Center of Nanning Customs, Nanning 530021, People's Republic of China. 
Lobata. Therefore, this study intends to establish a rapid and accurate method for the identification of biogenic Amines in Pueraria Lobata.

There were some analytical methods for the detection of biogenic amines such as GC-MS/MS [20], ion chromatography [21, 22], HPLC ([5, 23, 24], TLC [14], and HPLC-MS/MS methods $[2,17])$, while the pre-processing steps of those methods were relatively complex and difficult to operate. On the one hand, most of the reported pretreatment methods are suitable for high protein foods, but not for starchy root samples such as Pueraria Lobata. On the other hand, there are few reports on the simultaneous determination of multi-biogenic amines, but the determination period of multi-biogenic amines was over half an hour, so the results cannot be displayed quickly. In this experiment, a method for the identification and simultaneous detection of the nine biogenic amines in pueraria lobata by UPLC with precolumn derivatization had been established. It could be used columns with small particle sizes to achieve better separation and shorter analysis time through UPLC system. Besides, it could be simplified the pre-processing steps and provided the quick detection method for multi-biogenic amines in pueraria lobata. Through the established method, it was verified that there are natural biogenic amines in pueraria lobata.

\section{Material and methods}

\subsection{Materials}

All solvents were of analytical reagent grade except for special instructions. (Millipore, USA) Deionized water by Milli-Q Integral 10 ultra-pure water machine (Milli-Q; Millipore, Bedford, MA, USA) was used through the study. Standard of putrescine, tyramine, histamine, spermine, spermidine, phenethylamine, octopamine, cadaverine, were purchased from Dr. Ehrenstorfer (Germany), tryptamine was purchased from ANPEL (Shanghai, China). The standards of putrescine, histamine, spermine and spermidine were of $99.0 \%$ purity; tyramine was of $99.5 \%$, phenylethylamine was of $97.5 \%$, octopamine was of $96.5 \%$, cadaverine was of $99.0 \%$, tryptamine was of $98.0 \%$.

Dansyl chloride was purchased from ANPEL (Shanghai, China) with purity of $98.0 \%$. Acetonitrile, acetone and ammonium acetate with chromatographic purity were purchased from TEDIA Company (TEDIA, USA). Sodium bicarbonate, hydrochloric acid, perchloric acid and ammonia water were grade AR from Chemical Reagent Co., Ltd.

The plant samples of pueraria lobata were purchased from Guangxi medicine markets.

\subsubsection{Preparation of standard solutions}

Biogenic amine single stock solutions: $50 \mathrm{mg}$ of standard of putrescine, tyramine, histamine, spermine, spermidine, phenethylamine, octopamine, cadaverine, and tryptamine was weighed accurately and put into $50 \mathrm{~mL}$ volumetric flasks respectively; $0.1 \mathrm{~mol} / \mathrm{L}$ hydrochloric acid was put into the volumetric flasks to bring to volume; The materials in the volumetric flasks were prepared into $1.0 \mathrm{mg} / \mathrm{mL}$ standard stock solutions and then stored in a refrigerator at $4{ }^{\circ} \mathrm{C}$.

Mixed stock solution of nine standard biogenic amines: $1.00 \mathrm{~mL}$ of each biogenic amine single-standard stock solution was pipetted and mixed in the same $10 \mathrm{~mL}$ volumetric flask, $0.1 \mathrm{~mol} / \mathrm{L}$ hydrochloric acid was put into the volumetric flask to bring to volume and then the materials in the volumetric flask were prepared into $100 \mu \mathrm{g} / \mathrm{mL}$ biogenic amine mixed stock solution.

Mixed working solution of nine standard biogenic amines: According to the experiment needs, the biogenic amines mixed solution was diluted by water and prepared into the biogenic amine mixed-standard working solutions of different concentrations.

\subsubsection{The derivatization of biogenic amines standard solution}

Appropriate amount of biogenic amine mixed working solution was pipetted into a $15 \mathrm{~mL}$ centrifuge tube, and then $1.5 \mathrm{~mL}$ of saturated sodium carbonate solution and $1 \mathrm{~mL}$ of dansyl chloride solution $(10 \mathrm{mg} / \mathrm{mL}$, dissolved in acetone) were added. After the tube being covered, it was placed and shaken in a temperature-controlled shaker at $40{ }^{\circ} \mathrm{C}$ without light for an hour. Then, $100 \mu \mathrm{L}$ of ammonia was added to terminate the reaction. The volume was adjusted to $5 \mathrm{~mL}$ with acetonitrile. The centrifugation was performed at $4000 \mathrm{r} / \mathrm{min}$ for $5 \mathrm{~min}$. Finally, the supernatant was filtered through a $0.22 \mu \mathrm{m}$ filter for liquid chromatography.

\subsection{Preparation of the samples for UPLC-PDA analysis}

An amount of $2.00 \pm 0.01 \mathrm{~g}$ of homogenized sample (by FLUKO FA25 dispersing homogenizer, FLUKO, Germany) was accurately weighed and placed into a $50 \mathrm{~mL}$ centrifuge tube, $20 \mathrm{~mL}$ of $0.4 \mathrm{~mol} / \mathrm{L}$ perchloric acid was added into the tube. Then, the sample was homogenized for two minutes and centrifuged by SIGMA3-18 K high-speed refrigerated centrifuge (SIGMA, Germany) at $10000 \mathrm{r} / \mathrm{min}$ for $10 \mathrm{~min}$ at $4{ }^{\circ} \mathrm{C} .1 .00 \mathrm{~mL}$ of supernatant was pipetted into a $15 \mathrm{~mL}$ centrifuge tube. Then, $1.50 \mathrm{~mL}$ of saturated sodium bicarbonate solution and $1.00 \mathrm{~mL}$ of dansyl chloride 
solution (10 mg/mL, dissolved in acetone) were added. After being covered, the tube was placed and shaken in a temperature-controlled shaker (ALT-032R intelligent temperature-controlled shaker, Shanghai Kanxin Instrument Equipment Co., Ltd.) at $40{ }^{\circ} \mathrm{C}$ avoiding light for an hour. Then, $100 \mu \mathrm{L}$ of ammonia was added to stop the derivative reaction. The reaction liquid was diluted to $5.00 \mathrm{~mL}$ by acetonitrile and centrifuged at $4000 \mathrm{r} / \mathrm{min}$ for $5 \mathrm{~min}$. Finally, the supernatant was filtered through a $0.22 \mu \mathrm{m}$ filter for ultra performance liquid chromatography analysis.

\subsection{UPLC analysis and calculations}

The analyses were performed using Waters Acquity UPLC system (Waters, USA) comprising a Binary Solvent Manager pump, Sample Manager and PDA (Photo-Diode Array) detector. A UPLC BEH C18 $(1.7 \mu \mathrm{m}, 2.1 \mathrm{~mm} \times 100 \mathrm{~mm})$ (Waters, USA) column was used for separating the nine biogenic amines. Temperature of the UPLC column was set at $35^{\circ} \mathrm{C}$. The mobile phase was pumped at a rate of $0.4 \mathrm{~mL} / \mathrm{min}$. The gradient elution performed was shown as Table 1, which mobile phase A for $5 \mathrm{mmol} / \mathrm{L}$ ammonium acetate, mobile phase $B$ for acetonitrile. The sample volume injected was $5 \mu \mathrm{L}$. The spectra of the nine biogenic amines were obtained from detecting at the wavelength of $254 \mathrm{~nm}$.

Calibration standards were prepared at concentrations of $0.1,0.5,1.0,5.0,10.0$, and $20.0 \mu \mathrm{g} / \mathrm{mL}$ of the mixed standard solution of the nine compounds, and injected in triplicate. Calculations were based on the standard calibration curves obtained by plotting the peak areas $(\mathrm{Y})$ of multi-biogenic amines versus their concentrations $(X)$ in methanol.

\subsection{Method validation}

The method was validated with reference to the implemented validation procedure for analytical methods as described in the EU Commission Decision 2002/657/EC under Council Directive 96/23/EC [1] and in Commission recommendation of 10 January 2003 concerning a coordinated programmer for the official control of foodstuffs

Table 1 Gradient elution program for UPLC

\begin{tabular}{lll}
\hline Time (min) & $\begin{array}{l}\text { Mobile phase A propor- } \\
\text { tion (\%) }\end{array}$ & $\begin{array}{l}\text { Mobile phase B } \\
\text { proportion (\%) }\end{array}$ \\
\hline 0.00 & 45 & 55 \\
5.00 & 5 & 95 \\
6.00 & 5 & 95 \\
7.00 & 45 & 55 \\
8.00 & 45 & 55 \\
\hline
\end{tabular}

for 2003 [3]. It is worth to note that the method for determination of multi-biogenic amines was not belonged to residues analysis under the EU system, so it was just for a reference to the validation process of the established method with the directive.

\subsubsection{Recovery}

Three sets, each of six, of Pueraria lobata samples were spiked with $20,50,100 \mathrm{mg} / \mathrm{kg}$ of the mixed standards, respectively, and analyzed.

The absolute recovery was calculated by comparing the peak areas from the standard calibration curves of multi-biogenic amines with the peak areas obtained after extraction of the same amounts of the compounds from spiked samples. Percentage recovery was calculated as 100 times amount of subtracting the amount contained in the sample from the amount determined.

\subsubsection{Repeatability}

Three sets, each of six samples that have been spiked with multi-biogenic amines at the concentrations described above, respectively, were analyzed on the same day with the same instrument and operator. The overall mean concentrations and RSD were calculated.

Three sets, each of six samples that have been spiked with multi-biogenic amines at the concentrations described above, respectively, were analyzed on different days with the same instrument and operator. The overall mean concentrations and RSD were calculated.

\section{Results and discussion}

\subsection{The optimization of preparation}

Previous experiments $[4,25,26]$ showed that the sample preparation was relatively complicated, including $n$-hexane degreasing, liquid-liquid extraction, $\mathrm{pH}$ adjustment, nitrogen blowing and other steps and requires many organic solvents, which was harmful to the health of the experimental personnel. In this experiment, the pretreatment steps were optimized and the samples were directly extracted and then derivatized. The extraction effects of water, perchloric acid solution, hydrochloric acid solution, methanol and acetonitrile were investigated. The results showed that perchloric acid solution had the best extraction efficiency, and achieved the highest overall recovery rate of the 9 kinds of biogenic amines than other extract solvents.

Since most biogenic amines were not UV-chromophoric groups, they needed to be derivatized before 
they could be detected. In this method, it had been compared the two frequently-used derivatizing reagents, o-phthalaldehyde (OPA) and dansyl chloride (Dns-Cl). The result showed that dansyl chloride had better derivatization effect, in which the derivatization conditions were easy to control, the product after derivatization was stable and the reproducibility was good. Although the reaction time of o-phthalaldehyde was short, the product after derivatization was unstable and had poor reproducibility.

All the above led to the conditions for the sample preparation described in Sect. 2.2.
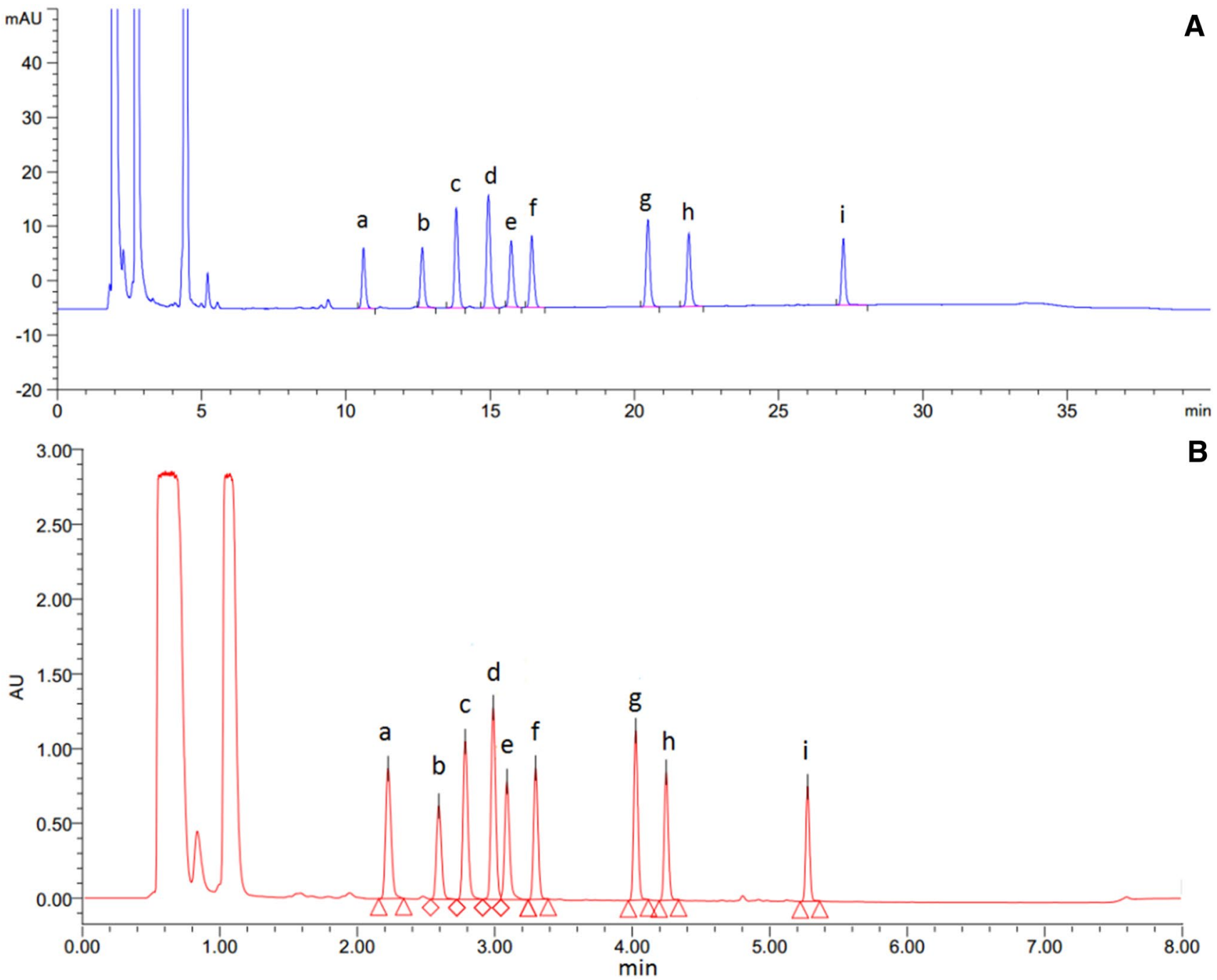

Fig. 1 Comparison of Chromatographic Charts of Nine Biogenic Amines with Concentrations of $0.2 \mu \mathrm{g} / \mathrm{mL}$ Using HPLC (A) and UPLC (B). A: $a$ tryptamine (10.613 min); $b$. phenethylamine (12.647 $\mathrm{min}) ; c$. putrescine (13.830 $\mathrm{min}) ; d$. cadaverine (14.941 min); e. histamine (15.730 $\mathrm{min}) ; f$. octopamine (16.446 $\mathrm{min}) ; g$. tyramine

\subsection{The optimization of chromatographic conditions}

\subsubsection{Comparison of speed and performance between UPLC and HPLC}

Agilent 1260 HPLC instrument was used to detect the chromatographic conditions according to the Chinese National Standard GB 5009.208-2016 [4]. The HPLC chart was shown in Fig. 1 a.

From Fig. 1a, it showed that it had been taken about $30 \mathrm{~min}$ for the peaks of the 9 biogenic amines to appear completely, and it taken more than $35 \mathrm{~min}$ to complete one injection. tryptamine $(2.269 \mathrm{~min}) ; b$. phenethylamine $(2.635 \mathrm{~min}) ; c$. putrescine (2.835 min); $d$. cadaverine (3.039 min); e. histamine (3.139 min); $f$. octopamine (3.349 $\mathrm{min}) ; g$. tyramine $(4.073 \mathrm{~min}) ; h$. spermidine (4.295 $\mathrm{min})$; i. spermine (5.329 min) 
However, the UPLC platform was characterized by its high pressure resistance and small particle size, which enabled faster analysis, better separation and high sensitivity, and doubled detection efficiency. It took only about 5 min for the peaks of the 9 types of biogenic amines to fully emerge (See Fig. 1b). It took only 8 min to complete a sample injection with good peak shape and the resolution, and it was not disturbed by the heterochromatic peaks. It was suitable for rapid detection of large batches of samples. The chromatogram of the standard solution with concentration of $0.2 \mu \mathrm{g} / \mathrm{mL}$ was shown in Fig. 1 b. From Fig. 1 b, it could be seen that the peaks of the 9 biogenic amines were sharp and symmetrical with no interference of heterochromatic peaks, and could be completely separated.

The result of the comparison led to the conditions for the analysis described in Sect. 2.3.

\subsubsection{The selection of the mobile phases}

Three most widely used mobile phase systems including acetonitrile-water, acetonitrile- $10 \mathrm{mmol} / \mathrm{L}$ ammonium acetate buffer solution and methanol-water were selected as comparisons. It was found that when methanol-water was used as the mobile phase, the chromatographic peaks of phenylethylamine and putrescine, amines and tyramine could not be completely separated. When acetonitrile-water was used as the mobile phase, the retention times of cadaverine and histamine were not stable enough, and the two components could not be completely separated due to the shift of retention time, which affected the accuracy of the quantization. When using the mobile phase system of acetonitrile-ammonium acetate buffer salt solution, each target peak had better and symmetrical peak shape, with no tailing phenomenon, no disturbance of hetero peaks, complete separation of components and stable retention time. Therefore, acetonitrile-10 $\mathrm{mmol} / \mathrm{L}$ ammonium acetate buffer salt solution was used as the mobile phase system.

\subsubsection{The selection of analysis columns}

An Acquity UPLC BEH C18 $(1.7 \mu \mathrm{m}, 2.1 \times 100 \mathrm{~mm})$ column and an Acquity UPLC BEH Shield RP18 $(1.7 \mu \mathrm{m}$, $2.1 \times 100 \mathrm{~mm}$ ) column were investigated. When using the
Acquity UPLC BEH Shield RP18 column, the time taken for the peaks of the 9 biogenic amines to complete was shorter than compared to Acquity UPLC BEH C 18 column, but cadaverine and histamine could not be completely separated. And when using the Acquity UPLC BEH C18 column, the peak response values of the 9 the biogenic amines were higher than compared to the Acquity UPLC BEH Shield RP18 column. Therefore, the Acquity UPLC BEH C18 column was selected for this method.

\subsection{Linear range and limit of quantitation}

Biogenic amine mixed-standard solutions with concentrations of $0.1,0.5,1.0,5.0,10.0$, and $20.0 \mu \mathrm{g} / \mathrm{mL}$ for each component were prepared for detection. The standard curves were made, with the concentrations of biogenic amines mixed-standard solution as abscissa and peaks area as ordinate. In the range of $0.1-20 \mu \mathrm{g} / \mathrm{mL}$, the concentrations of the 9 biogenic amines and the corresponding peaks area were shown good liners relationship $(r>0.999)$ within the tested range.

It showed that limits of quantitation for the nine biogenic amines in Pueraria Lobata samples were all at concentrations of $10.0 \mathrm{mg} / \mathrm{kg}$.

\subsection{The recovery rate of standard addition and precision}

The method was further tested by applying the extraction procedure to analysis of spiked Pueraria Lobata samples for verification of the limit of quantitation. Mixed standard solutions of 9 biogenic amines were spiked to Pueraria Lobata samples at three different concentrations ranging from 20 to $100 \mathrm{mg} / \mathrm{kg}$.

The detection was performed with this method. And recovery rate of standard addition and precision experiments were performed. It were shown in Table 2 that the 9 biogenic amines spiked with the concentrations of 20 , 50 , and $100 \mathrm{mg} / \mathrm{kg}$, the average recovery rates range were from 75.6 to $97.2 \%(n=6)$, with a relative standard deviation (RSD) of $0.12-5.32 \%(n=6)$, meeting the test method's requirements for recovery rate and precision. Repeat the test for 7 days of the same samples, the relative standard deviation of 9 kinds of biogenic amines were less than

Table 2 Average recovery rates and precision of nine biogenic amines added in Pueraria Lobata samples $(n=6)$

\begin{tabular}{|c|c|c|c|c|c|c|c|c|c|}
\hline \multirow{2}{*}{$\begin{array}{l}\text { Addition(mg/ } \\
\mathrm{kg} \text { ) }\end{array}$} & \multicolumn{9}{|c|}{ Average recovery rates \pm (relative standard deviation/\%) } \\
\hline & Try & Phe & Put & Cad & His & $\mathrm{Oa}$ & Tyr & Spd & Spm \\
\hline 20 & $77.9 \pm 0.51$ & $80.6 \pm 0.26$ & $82.3 \pm 0.12$ & $84.6 \pm 2.71$ & $86.7 \pm 0.72$ & $83.9 \pm 0.44$ & $81.3 \pm 1.31$ & $79.2 \pm 0.91$ & $75.6 \pm 5.32$ \\
\hline 50 & $86.7 \pm 0.76$ & $86.2 \pm 1.03$ & $90.5 \pm 2.37$ & $87.2 \pm 2.71$ & $88.2 \pm 0.61$ & $90.6 \pm 2.93$ & $86.2 \pm 0.34$ & $85.7 \pm 1.14$ & $86.5 \pm 1.70$ \\
\hline 200 & $91.2 \pm 1.21$ & $93.5 \pm 0.65$ & $92.3 \pm 1.65$ & $94.1 \pm 1.42$ & $96.6 \pm 1.23$ & $89.1 \pm 1.68$ & $83.9 \pm 0.56$ & $87.7 \pm 2.51$ & $85.0 \pm 1.28$ \\
\hline
\end{tabular}


$5.2 \%$, and the results showed that it was good stability for the method. The chromatogram of Pueraria Lobata sample and chromatogram of additional standards were shown in Figs. 2 and 3.

\subsection{Actual sample test results}

A total of 12 samples of Pueraria Lobata from the different place were analyzed by the method being developed by ourselves. The results of the positive samples are shown in Table 3.

It was found that phenylethylamine, putrescine, cadaveric amine, spermidine, spermine and histamine were detected in the root of Pueraria lobata. The content ranged from 9.79 to $125 \mathrm{mg} / \mathrm{kg}$. This is similar to the results of determination of biogenic amines in Evodia rutaecarpa by Sun et al. [13]. Pueraria lobata and Evodia rutaecarpa are both Chinese medicinal plants, but Pueraria lobata can also

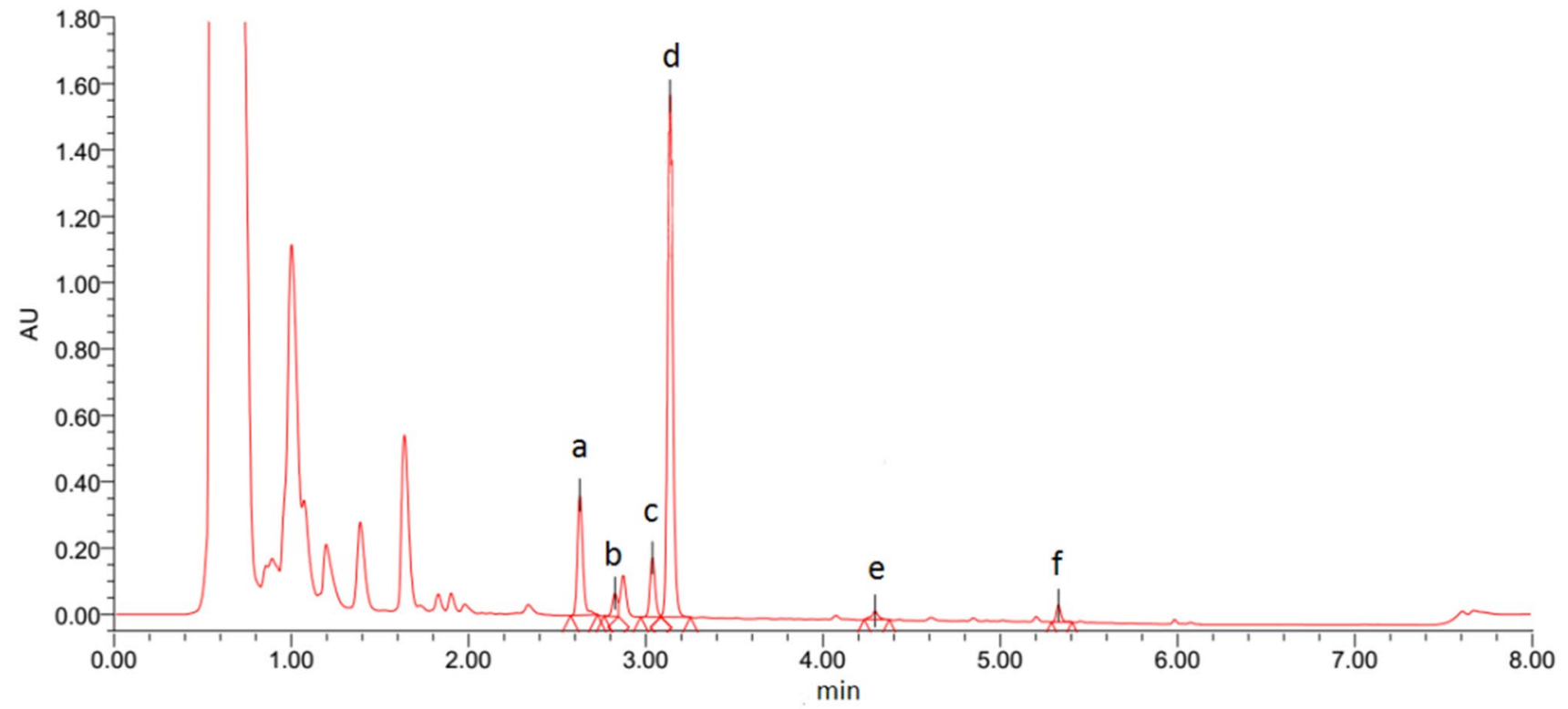

Fig. 2 UPLC chromatogram of the fresh Pueraria Lobata sample with nature biogenic amines. Note: $a$. phenethylamine (2.628 min); $b$. putrescine (2.826 min); c. cadaverine (3.037 min); $d$. histamine (3.136 min); e. spermidine (4.293 min); f. spermine (5.327 min)

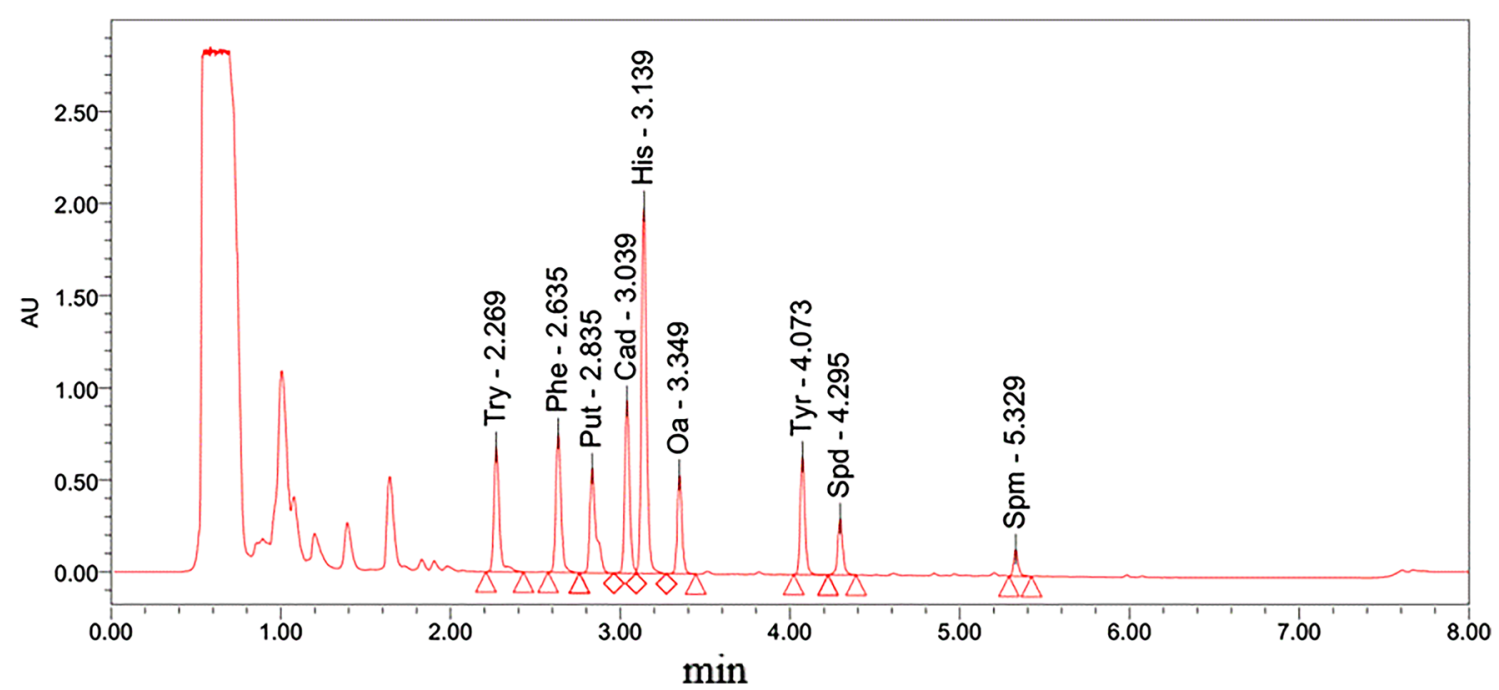

Fig. 3 UPLC chromatogram of fresh Pueraria Lobata sample added with Nine biogenic amines with concentrations of $200 \mathrm{mg} / \mathrm{kg}$. Note: $a$. tryptamine $(2.269 \mathrm{~min}) ; b$. phenethylamine $(2.635 \mathrm{~min}) ;$ c. putres- cine (2.835 min); $d$. cadaverine (3.039 min); e. histamine (3.139 min); $f$. octopamine (3.349 $\mathrm{min}) ; g$. tyramine $(4.073 \mathrm{~min}) ; h$. spermidine (4.295 $\mathrm{min})$; i. spermine (5.329 $\mathrm{min})$

\section{SN Applied Sciences}


Table 3 Biogenic amines contents of Pueraria Lobata samples from different origin place $(\mathrm{mg} / \mathrm{kg})(\mathrm{n}=6)$

\begin{tabular}{|c|c|c|c|c|c|c|c|c|c|}
\hline Sample & Try & Phe & Put & Cad & His & Tyr & Spd & Spm & \\
\hline $1 \#$ & \multicolumn{2}{|c|}{$23.9 \pm 0.0215 \pm 0.03$} & $13.4 \pm 0.02$ & $11 \pm 0.16$ & / & $9.76 \pm 0.52$ & $12.8 \pm 0.24$ & $18.5 \pm 0.06$ & Guigang \\
\hline $2 \#$ & $13 \pm 0.06$ & $/^{*}$ & $12.4 \pm 0.14$ & / & / & / & / & $74.2 \pm 1.28$ & Wuzhou \\
\hline $3 \#$ & \multicolumn{2}{|c|}{$16.7 \pm 0.59 /$} & / & / & / & / & $14.6 \pm 0.49$ & / & Hunan \\
\hline $4 \#$ & \multicolumn{2}{|c|}{$52.8 \pm 0.6710 .2 \pm 0.81$} & $30.9 \pm 0.92$ & / & / & / & $17.8 \pm 0.47$ & / & Guiping \\
\hline $5 \#$ & \multicolumn{2}{|c|}{$63.7 \pm 0.8111 .3 \pm 0.43$} & $71.2 \pm 1.29$ & $17.7 \pm 0.47$ & / & / & $26.6 \pm 0.64$ & / & Guiping \\
\hline $6 \#$ & $114 \pm 2.16$ & $40.6 \pm 0.92$ & $72.5 \pm 0.71$ & $17.7 \pm 0.53$ & / & $9.1 \pm 0.06$ & $35.3 \pm 0.43$ & / & Dabie Shan \\
\hline 7\# & $99 \pm 1.02$ & $76.9 \pm 0.93$ & $125 \pm 2.64$ & $54.1 \pm 0.39$ & $12.3 \pm 0.24$ & $59.9 \pm 0.94$ & $13.9 \pm 0.32$ & $15.8 \pm 0.24$ & Zhangjiajie \\
\hline $8 \#$ & \multicolumn{2}{|c|}{$16.4 \pm 0.29 /$} & $7.78 \pm 0.09$ & / & / & / & / & / & Wuzhou \\
\hline 9\# & $65 \pm 1.21$ & $23.1 \pm 0.94$ & $44.5 \pm 0.97$ & $26 \pm 0.69$ & $14.1 \pm 0.52$ & / & $28.4 \pm 0.63$ & / & Yulin \\
\hline $10 \#$ & $60 \pm 1.18$ & $25.6 \pm 0.89$ & $56.1 \pm 0.94$ & / & / & / & $22.9 \pm 0.63$ & / & Beihai \\
\hline $11 \#$ & \multicolumn{2}{|c|}{$83.8 \pm 1.2320 .2 \pm 0.72$} & $57.8 \pm 0.96$ & $12.5 \pm 0.43$ & $9.79 \pm 0.39$ & / & $16.1 \pm 0.41$ & / & Wuzhou \\
\hline $12 \#$ & \multicolumn{2}{|c|}{$27.8 \pm 0.63 /$} & $18.9 \pm 0.52$ & / & / & / & $10.3 \pm 0.13$ & / & Wuzhou \\
\hline
\end{tabular}

* "/" means not detected

be eaten. However, there are differences in species and biogenic amines content. The results of contents of nine biogenic amines in Pueraria lobata were shown in Table 3.

\section{Conclusion}

A UPLC method has been firstly developed for the simultaneous detection and quantification of nine 9 biogenic amines in pueraria lobata. It was simplified of the sample pretreatment process and replaced some complicated extraction steps reported in previous literatures by the method. Good linearity had been demonstrated over the concentrations ranged from 10 to $200 \mathrm{mg} / \mathrm{kg}$ in pueraria lobata. Accuracy and precision at the spiking levels for the method were according with the requirement of analysis in food producing. A sample could be detected within shortest period under the established chromatographic conditions. The detection limits of the 9 biogenic amines were all at $10 \mathrm{mg} / \mathrm{kg}$. Precision experiments showed that this method was reproducible. Most of all, a simple, fast, accurate and reliable method had been established, which could be used to identify and quantify for 9 biogenic amines in Pueraria lobata. By the established method, it was found for the first time that there are different kinds of biogenic amines in Pueraria lobata, and the specific types and contents of biogenic amines varied according to the variety and origin of the specific of Pueraria lobata. This study will provide some ideas and help for the study of Pueraria lobata's pharmacodynamic mechanism and so on.

Acknowledgements The authors thank the Ministry of Science and Technology of the People's Republic of China and Special Fund for Basic Scientific Research Operation Expenses of central public welfare scientific research institutes of CAIQ for the financial support, which enabled this work to be carried out. The number of the project was
2017YFF0211000 and 2020JK007. And we thank all those who helped us to establish this method and find maybe useful biogenic amines in Pueraria Lobata which is one of medicinal and edible homologous plants.

\section{Compliance with ethical standards}

Conflict of interest The authors declare that they have no conflict of interest.

\section{References}

1. Commission Decision of 12 August 2002 implementing Council Directive 96/23/EC concerning the performance of analytical methods and the interpretation of results (2002/657/EC), Official J Eur Commun L221 (2002) 8

2. Ding T, Lv C, Liu H et al (2014) Determination of eight biogenic amines in red wines by liquid chromatography-quadrupole/ electrostatic field orbit trap mass spectrometry. J Chin Instrum Anal 33(1):27-32

3. European Commission (EC) (2003) Commission recommendation of 10 January 2003 concerning a coordinated programmer for the official control of foodstuffs for 2003 (2003/10/EC).

4. GB 5009.208-2016. Chinese National Standard of Food Safety of Determination of biogenic amine in foods.

5. Hu Y, Huang ZY (2012) Column before derivative HPLC determination of many kinds of biogenic amines in fish at the same time and its change rule. J Food Sci Technol 12(11):142-147

6. Kalac P, Krausova P (2005) A review of dietary polyamines: formation, implications for growth and health and occurrence in foods. Food Chem 77:349-351

7. Lange J, Thomas K, Wittmann C (2002) Comparison of a capillary electrophoresis method with high-performance liquid chromatography for the determination of biogenic amines in various food samples. J Chromatogr B 779:229-239

8. Liu J, Ren J, Sun KJ (2013) Safety of biogenic amines in foods. Chin J Food Sci 34(5):322-326

9. Silla Santos MH (1996) Biogenic amines: their importance in foods. Int J Food Microbiol 29:213-231 
10. Shalaby AR (1996) Significance of biogenic amines to food safety and human health. Food Res Int 29:675-690

11. Stratton JE, Hutkins RW, Taylors SL (1991) Biogenic amines in cheese and other fermented foods: a review. J Food Protect 54:460-470

12. Stute $R$, Petridis $K$, Steinhart $H$ et al (2002) Biogenic amine in fish and soy sauces. Eur Food Res Technol 215(2):101-107

13. Sun YN, Zhang N, Wang CL (2014) Identification and determination of biogenic amines in Evodia Rutaecarpa (Juss.) Benth by high performance liquid chromatography. Chin J Anal Chem 42(2):273-277

14. Shalaby AR (1999) Simple rapid and valid thin layer chromatographic method for determining biogenic amines in foods. Food Chem 65(1):117-121

15. Tassoni A, Germana MA, Bagni N (2004) Free and conjugated polyamine content in Citrus sinensis Osbeck, cultivar brasilino N.L. 92, a Navel orange, at different maturation stages. Food Chem 87:537-541

16. Vinci G, Antonelli ML (2002) Biogenic amines: quality index of freshness in red and white meat. Food Control 13:S19-524

17. Wang GQ, Yu JS, Hu J et al (2016) Progress in research on biogenic amines in foods. Chin J Food Sci 37(1):269-278

18. Wu YH, Zhou S, Xu GM (2013) Determination of eight biogenic amines in animal-derived foodstuffs by high performance liquid chromatography-tandem mass spectrometry without derivatization. Chin J Chromatogr 31(2):111-116

19. U.S. Food and Drug Administration. CPG Sec. 540.525 Decomposition and histamine raw, frozen tuna and mahi-Mahi; canned tuna; and related species, revised compliance policy guide, availability.
20. Wen YZ, Fan WL, Xu Y (2013) Identification of a variety of biogenic amines by GC-MS method in China's liquor. Chin J Liquor Making 140(1):38-41

21. Zhao XY, Jiao X, Xia M et al (2009) Determination of five biogenic amines in source water by ion chromatography. Chin J Chromatogr 27(4):505-508

22. Zhou Y, Wang PY, Zhao H et al (2014) Determination of biogenic amines in frozen meat products by high performance ion-exchange chromatography-electrochemical. Chin J Food Ind 35(5):238-241

23. Zhai HL, Yang XQ, Hao SX et al (2011) Optimization of operating conditions for HPLC determination of biogenic amines. Chin J Food Sci 32(18):180-184

24. Zhang N, Wang CL, Hong BL et al (2015) Identification and determination of biogenic amines in Ephedrae Herba by RPHPLC with precolumn derivatization. Chin J Pharmaceut Anal 35(3):389-395

25. Zhang Y, Wu GH, Liu WB et al (2012) Simultaneous determination of 8 biogenic amine residues in aquatic products by ultrahigh performance liquid chromatography. Chin J Food Sci 33(18):181-185

26. Zhou Y, Zhao MM, Zhao HF (2012) Simultaneous determination of 8 kinds of biogenic amines in soy sauce by HPLC. Chin J Modern Food Sci Technol 28(5):570-573

Publisher's Note Springer Nature remains neutral with regard to jurisdictional claims in published maps and institutional affiliations. 